\title{
РАСОВОЕ ПРОФИЛИРОВАНИЕ: ИСТОРИЯ И СОВРЕМЕННОЕ СОСТОЯНИЕ ИССЛЕДОВАНИЙ
}

\author{
КСЕНИЯ ГРИГОРЬЕВА
}

\begin{abstract}
Немногочисленные исследования расового профилирования в России подтвердили существование этой проблемы. Однако её разработка, начатая в середине 2000-х годов, до настоящего времени продвинулась незначительно. Русскоязычный читатель практически не знаком с теоретическими и методологическими подходами к изучению расового профилирования, разработанными за рубежом. Эта статья призвана восполнить недостаток информации о зарубежных исследованиях расового профилирования и является первым обзором литературы по этой теме на русском языке. В статье рассматривается история изучения расовой дискриминаџии в системе уголовного правосудия в англоязычном научном мире, анализируется выделение расового профилирования в отдельную область исследований в начале 1990-х годов и ее дальнейшее развитие в 2000-х годах. Представлень основные методологические подходы к изучению расового профилирования, их возможности и ограничения. Рассмотрены ключевые теоретические подходы к пониманию расового неравенства в правоохранительной деятельности. В заключение с учетом накопленного опыта приводится ряд рекомендаций по планированию будущих исследований. Обращается внимание на необходимость более четкого разграничения между преднамеренным расовым профилированием, санкционированным государством; политикой отдельных полицейских управлений; личной иниџиативой сотрудников полиции и статистической дискриминацией, отражающей социальное неравенство. При проведении количественных исследований рекомендуется использовать несколько источников данных и несколько аналитических методов. Подчеркивается необходимость избегать поспешности и предвзятости в выводах. При интерпретащии полученных эмпирических данных рекомендуется учитывать все возможные концептуальные объяснения, включая расово нейтральнье.
\end{abstract}

Ключевые слова: расовая дискриминаџия, уголовная юстиция, расовое профилирование, проблема знаменателя, индивидуальная и институцииоальная расовая предвзятость, осознанные $и$ неосознанные расовые предрассудки.

Расовая дискриминация в правоохранительной системе - международно признанная проблема, привлекающая внимание исследователей по всему миру в течение многих лет. Расовое профилирование ${ }^{1}$, являющееся одной из её разновидностей, - значительно более недавнее направление общественно-политических дискуссий и научных изысканий. Но, несмотря на относительную непродолжительность своего существования, эта исследовательская область стремительно развивается. Возникнув в первой половине 1990-х годов в США, она быстро приобрела популярность в англоязычном научном мире, а в начале 2000-х годов - и за его пределами.

КСЕНИЯ СЕРГЕЕВНА ГРИГОРЬЕВА (kseniagrigoryeva@yandex.ru), ИНСТИТУТ СОЦИОЛОГИИ ФНИСЦ РАН, Россия.

СТАТЬЯ ПОСТУПИЛА В РЕДАКЦИЮ В ОКТЯБРЕ 2019 Г.

${ }^{1}$ Расовое профилирование определяется как «использование правоохранительными органами, без объективных и разумных обоснований, таких признаков, как раса, цвет кожи, язык, религия, гражданство или национальное или этническое происхождение при контроле, слежении или проведении расследований» (ЕКРH 2007). 
В России первое количественное эмпирическое исследование расового профилирования было проведено в 2005 г. (Этнически избирательный... 2006). Вслед за ним в 2006-2008 гг. было осуществлено этнографическое исследование взаимодействия полиции и этнических меньшинств (Воронков, Гладарев, Сагитова 2011).

Результаты первого проекта отразили значимый этнический дисбаланс в полицейских остановках пассажиров московского метрополитена, результаты второго явную этническую дифференциацию в работе по меньшей мере некоторых сотрудников полиции Санкт-Петербурга и Казани, а также определенные, в основном косвенные, указания на существование этнически дискриминационных распоряжений полицейского руководства $^{2}$. Недавний анализ документальных источников подтвердил институциональный характер расового профилирования и выявил его распространение во многих российских регионах (Григорьева 2019). Тем не менее на сегодняшний день это явление остается мало исследованным в России.

За рубежом к настоящему моменту уже накоплен значительный объем эмпирических данных о расовом профилировании в разных странах мира, разработаны специфические теоретико-методологические подходы к его изучению и осмыслены их ограничения. К сожалению, российскому читателю они практически не известны.

Настоящая статья призвана восполнить эту лакуну и представляет собой первый углубленный обзор зарубежных исследований расового профилирования на русском языке.

\section{РАСОВАЯ ДИСКРИМИНАЦИЯ В СИСТЕМЕ УГОЛОВНОЙ ЮСТИЦИИ: ИССЛЕДОВАНИЯ 1950-80-Х ГОДОВ}

Изучение расового профилирования является одним из направлений исследования системы уголовного правосудия, имеющего богатую историю в англоязычном научном мире. Она началась в 50-е годы XX века, когда Американский фонд адвокатов при поддержке Фонда Форда осуществил первое большое эмпирическое исследование повседневной работы учреждений уголовной юстиции. Научная группа, находившаяся под влиянием подходов Чикагской социологической школы, сфокусировалась на наблюдении за тем, что в действительности происходило на улицах, в прокуратуре, судах и исправительных учреждениях. Результатом стало открытие ошеломляющих масштабов усмотрения на каждом этапе отправления уголовного правосудия. Полицейские, прокуроры и адвокаты, по свидетельствам наблюдателей, нередко не только игнорировали законодательные нормы, но и просто не знали их. В работе полиции повсеместно царил произвол, применялись незаконные аресты, умышленное жестокое обращение с подозреваемыми, в особенности с чернокожими. Вместе с тем полицейские зачастую чутко реагировали на

\footnotetext{
2 Было приведено всего два таких распоряжения: распоряжение начальника уголовной милиции УВД ЗАО г. Москвы о заведении оперативных дел на некоторые этнические группы и нашумевшее распоряжение ГУВД Санкт-Петербурга и Ленобласти о массовых проверках грузин. Однако авторы указывали на широкое распространение распоряжений, содержащих признаки этнической дискриминации, ссылаясь на свидетельства респондентов из числа сотрудников правоохранительных органов и правозащитных организаций.
} 
широкий круг социальных проблем, существующих в местном сообществе, выполняя миротворческую функцию и помогая регулировать мелкие споры, семейные и соседские ссоры и др. (Walker 1992). Исследование продемонстрировало неадекватность прежнего взгляда на уголовную юстицию ${ }^{3}$ и фактически привело к формированию новой академической дисциплины. Стали доминировать представления о сложности системы уголовного правосудия, её пронизанности дискреционными решениями ${ }^{4}$, взаимозависимости работы входящих в неё учреждений, слабости формального контроля и действия правовых норм.

Особое место в исследованиях уголовной юстиции очень скоро заняло изучение полиции. Помимо того, что она оказалась в центре внимания участников проекта Американского фонда адвокатов, многие из которых впоследствии были признаны ведущими экспертами в области уголовного правосудия и продолжали работу в данном направлении, интерес к полиции был вызван рядом неакадемических обстоятельств. В частности, этому способствовала проблематизация преступности в ходе американских президентских предвыборных кампаний 60-х годов, а также ряд резонансных решений Верховного суда США, направленных на защиту граждан от полицейского усмотрения 5 .

Наиболее популярным методом изучения полиции со времени реализации проекта Американского фонда адвокатов стало включенное наблюдение. Публикации результатов таких этнографических исследований, вышедшие из печати в 60-70-е годы ${ }^{6}$, впоследствии приобрели статус классических (Remington 1960; LaFave 1962; Goldstein 1963; Skolnick 1966; Bittner 1970). В центре анализа, как правило, находились общие проблемы полицейской деятельности, тем не менее некоторые авторы рассматривали и вопросы расовой предвзятости. Так, Дж. Сколник посвятил этому один из разделов своей монографии «Правосудие без суда: правоохранительная деятельность в демократическом обществе» (Skolnick 1966). Высказанные им идеи о связи расовых предрассудков с полицейской субкультурой, учете полицейскими не только расовых, но также классовых и гендерных характеристик подозреваемых, важности ситуативных факторов, ограничивающих влияние расовой предвзятости в реальной правоохранительной

\footnotetext{
${ }^{3}$ Он характеризовался, с одной стороны, реформистской идеологией, а с другой стороны, своеобразным методологическим подходом, не выходившим за рамки анализа официальных данных. Основной проблемой уголовного правосудия считался малый процент дел, окончившихся обвинительными приговорами. Причины этого усматривались в политическом вмешательстве, низкой квалификации специалистов, нехватке данных и ресурсов. Предполагалось, что необходимо провести реформу системы уголовного правосудия с целью пресечения политического манипулирования и коррупции, заменить плохих специалистов хорошими, вооружить их необходимой информацией и ресурсами (Walker 1992).

${ }^{4}$ Причем эта дискреционность стала рассматриваться амбивалентно, как нечто, что обладает не только и не столько негативным характером, сколько имеет позитивные функции в решении проблем локальных сообществ.

${ }^{5}$ Решение по делу Мапп против Огайо 1961 г., запретившее использовать улики, полученные незаконным путем, в суде; решение по делу Эскобедо против Иллинойса 1964 г., согласно которому подозреваемые в совершении уголовных преступлений имеют право на адвоката во время полицейских допросов; решение по делу Миранда против Аризоны 1966 г., установившее обязанность полицейских перед допросом сообщать подозреваемому, что он имеет право хранить молчание и требовать присутствия адвоката.

6 Часть из них являлась публикацией итогов проекта Американского фонда адвокатов, о котором говорилось выше.
} 
деятельности, оказали значительное влияние на последующие объяснительные модели специфики взаимодействия полиции и граждан из числа меньшинств.

Значимый вклад в исследование дискриминационного обращения полиции с меньшинствами внесли и основоположники так называемой «конфликтной перспективы» в изучении уголовной юстиции, вдохновленной марксистской теорией (Chambliss, Nagasawa 1969; Quinney 1970). Хотя они не ограничивались анализом расовой предвзятости полиции, поскольку в центре их интересов находились прежде всего вопросы классовой борьбы и использования правоохранительных органов как инструмента классового угнетения, сюжету расовой дискриминации в их работах также уделялось внимание. Примененный ими анализ официальных статистических данных об арестах в разбивке по расам для доказательства дискриминации афроамериканцев в системе уголовной юстиции впоследствии лег в основу исследования расовой предвзятости полиции. У. Чамблисс первым использовал концепцию самосбывающегося пророчества для объяснения того, как распространенные представления сотрудников правоохранительных органов о повышенной преступности чернокожих приводят к реальной перепредставленности последних в системе уголовного правосудия. Предложенные У. Чамблиссом и Р. Куинни объяснения дискриминационного обращения полицейских с меньшинствами (в том числе расовые предрассудки отдельных полицейских; повышенная «видимость» меньшинств; особенности их поведения, обусловленные культурными отличиями от большинства; распространенность в полицейском сообществе представлений об особой склонности некоторых этнических групп к преступной деятельности; «общественный запрос» белого большинства; намеренная государственная политика) во многом определили последующие направления исследований.

Повышению внимания к взаимодействию полиции и меньшинств в немалой степени способствовало Движение за гражданские права и расовые волнения в США, а также столкновения между полицией и чернокожей молодежью в Великобритании, распространившиеся в 80-е годы.

Исследования 70-80-х годов развивали идеи, заложенные в предшествующих работах. В частности, анализировалась связь расовой предвзятости полицейских и полицейской субкультуры (Colman, Gorman 1982); расовый дисбаланс в статистике допросов, обысков, арестов и тюремных заключений (Sherman 1980); распространенность представлений о повышенной преступности чернокожих среди сотрудников правоохранительных органов и общества в целом (Gilroy 1982).

Кроме того, в указанный период стали проводиться опросы о степени удовлетворенности полицейской деятельностью, которые использовались для анализа взаимоотношений полиции и общественности. Результаты этих опросов регулярно демонстрировали значимые различия в оценке правоохранительных органов белым и черным населением. Чернокожие (особенно молодые мужчины) были более пессимистичны в своих оценках полиции по сравнению с белыми (Lea 1986). 


\section{РАСОВОЕ ПРОФИЛИРОВАНИЕ: ВОЗНИКНОВЕНИЕ НОВОЙ ОБЛАСТИ ИССЛЕДОВАНИЯ}

Превращение расового профилирования в отдельную область исследования произошло в 1990-е годы и было связано с несколькими громкими судебными процессами в США. Первым из них стало дело «Нью-Джерси против Сото», в ходе которого 17 обвиняемых африканского происхождения заявили, что их аресты на одной из автомагистралей НьюДжерси, осуществленные в период с 1988 по 1991 г., явились результатом дискриминационного применения полицией правил дорожного движения (ПДД). Для проверки этого утверждения научной группой под руководством известного специалиста в области статистики и социальной психологии Дж. Ламберта в 1993 г. было осуществлено первое количественное эмпирическое исследование с целью установить наличие или отсутствие расового профилирования в работе дорожной полиции. На основе информации, содержащейся в полицейской отчетности, была создана база данных остановок и арестов за 35 дней, случайным образом выбранных в период с апреля 1988 г. по май 1991 г. Затем исследовательская команда провела наблюдение на четырех участках автомагистрали, чтобы установить расовый состав участников дорожного движения. Наконец, для разрешения вопроса о том, существуют ли различия в частоте нарушений правил дорожного движения белыми и черными водителями, участник научной группы Ф. Ласт совершил десять поездок по автомагистрали на своем автомобиле с использованием круиз-контроля, поддерживая максимально разрешенную скорость. В ходе поездок он фиксировал расу водителей и количество транспортных средств, которые его обгоняли, нарушая скоростной режим, а также совершение других нарушений ПДД. Всего таким образом было обследовано 2096 автомобилей, 98,1\% которых превысили допустимую скорость. При этом 15\% автомобилистов, совершивших то или иное нарушение, являлись чернокожими, что соответствовало доле черных водителей, участвовавших в дорожном движении. На основании этих наблюдений был сделан вывод, что манера вождения черных и белых автомобилистов не имеет существенных различий. Вместе с тем сравнение показателей остановок и арестов в разбивке по расам и соотношения черных и белых водителей, движущихся по автомагистрали, выявило статистически значимый расовый дисбаланс в работе полиции. Основываясь на этих данных, подкрепленных показаниями двух бывших полицейских, свидетельствовавших, что их обучали делать «профильные остановки» на основе расы, а также выявленным нежеланием руководителей государственной полиции принимать меры для пресечения расовой предвзятости в работе своих подчиненных, суд пришел к выводу, что полиция действительно осуществляла расовое профилирование (State v. Soto 1996). Решение суда вызвало значительный общественный резонанс, а Дж. Ламберт вскоре был приглашен в качестве эксперта по сходному процессу в штате Мэриленд («Уилкинс против полиции штата Мэриленд»), где было проведено новое исследование по той же методике, давшее аналогичные результаты. Дело было решено в пользу истца. На полицию штата Мэриленд были наложены обязательства официально запретить использование расового профилирования, провести программу переобучения своих сотрудников, вести систематический сбор информации об остановках, обысках и арестах автомобилистов, которая бы обеспечивала возможность установления наличия/отсутствия расового дисбаланса (Wilkins v. Maryland... 1994). После этих судебных решений иски против 
полиции по обвинению в расовом профилировании, неизменно сопровождавшиеся эмпирическими количественными исследованиями, стали подаваться и в других штатах.

На рубеже 1999-2000 годов общественно-политические дебаты вокруг расового профилирования в США приняли ожесточенный характер ${ }^{7}$. Репутации полиции был нанесен существенный урон. Власти отдельных штатов и руководители некоторых полицейских управлений, опасаясь возбуждения новых судебных разбирательств, начали обсуждать возможность добровольного сбора статистической информации о полицейских остановках и обысках. Только в 1999 г. соответствующие законодательные инициативы были выдвинуты в Арканзасе, Вирджинии, Иллинойсе, Калифорнии, Коннектикуте, Массачусетсе, Оклахоме, Северной Каролине, Огайо, Флориде и Южной Каролине (Нarris 1999).

Везде, где принималось положительное решение, к работе привлекались исследовательские группы, создавались базы данных, которые впоследствии анализировались другими учеными и научными коллективами. Разработка нового исследовательского направления стремительно развивалась, и к началу 2000 -х годов в США уже насчитывалось около трех десятков исследователей - специалистов в области изучения расового профилирования (Lamberth 2004).

Наивысшей точкой признания расового профилирования проблемой общенационального масштаба в США стало выступление Дж. Буша перед Конгрессом в феврале 2001 г., во время которого он заявил, что расовое профилирование является ошибкой и будет прекращено в Америке ${ }^{8}$. На международном уровне расовое профилирование было проблематизировано на Всемирной конференции по борьбе против расизма, расовой дискриминации, ксенофобии и связанной с ними нетерпимости в Дурбане, проходившей с 31 августа по 8 сентября 2001 г.

Теракт 11 сентября 2001 г. изменил направление общественно-политических дискуссий ${ }^{9}$, а объявление войны с терроризмом привело к распространению санкционированных государством практик расового профилирования в США, странахчленах ЕС и других странах мира. Это в свою очередь вызвало активизацию работы правозащитных организаций, создание новых международных рекомендаций по

\footnotetext{
${ }^{7}$ Сходные процессы наблюдались в Великобритании, где в конце 90-х годов также разразился кризис общественного доверия к правоохранительным органам, связанный с расследованием убийства чернокожего подростка С. Лоуренса, по результатам которого в 1999 г. был опубликован правительственный доклад сэра У. Макферсона. В докладе утверждалось наличие институционального расизма в английской полиции, а в качестве одной из причин недовольства меньшинств указывались их непропорциональные остановки и обыски. Инспекция Её Величества по делам полиции рекомендовала провести исследование данного вопроса. В соответствии с указанной рекомендацией и рекомендациями, содержавшимися в докладе У. Макферсона, английская полиция стала собирать статистические данные об остановках и обысках граждан с разбивкой по расе. В 2000 г. было проведено эмпирическое количественное исследование с целью установления наличия/отсутствия расового профилирования в работе правоохранительных органов (MVA, Miller 2000).

${ }^{8}$ George W. Bush on Racial Profiling (2001). URL: https://www.c-span.org/video/?c4524225/george-w-bushracial-profiling

${ }^{9}$ На смену практически единодушному осуждению расового профилирования пришла поддержка этой практики значительной частью населения (Jones 2001; Dearden 2017) и некоторыми экспертами (Colb 2004; Posner 2006).
} 
предотвращению расового профилирования (ЕКРН 2004; ЕКРН 2007) и дальнейший рост интереса научного сообщества к данному вопросу.

С начала 2000-х годов исследования расового профилирования стали приобретать всё большую популярность за пределами англоязычного научного мира, распространившись даже в тех странах, где проблемы этнической дискриминации в полицейской деятельности ранее не привлекали особого внимания (в том числе во Франции, Испании, Венгрии и Болгарии (см., например, (Goris, Jobard, Lévy 2009; Pap 2011; Miller et al. 2008)). В 2008 г. стартовало первое общеевропейское исследование по вопросам меньшинств и дискриминации, которое было нацелено, помимо прочего, на исследование расового профилирования (FRA 2010).

\section{МЕТОДОЛОГИЧЕСКИЕ ПРОБЛЕМЫ КОЛИЧЕСТВЕННЫХ ИССЛЕДОВАНИЙ РАСОВОГО ПРОФИЛИРОВАНИЯ}

Уже в начале 2000-х годов аналитиками были осознаны некоторые серьезные методологические проблемы, возникающие при проведении количественных исследований расового профилирования. Главной из них стала так называемая «проблема знаменателя» (MVA, Miller 2000; Walker 2001; Lamberth 2004), заключающаяся в том, что после сбора данных о количестве и процентном соотношении остановок и обысков граждан в разбивке по расам необходимо определить, является ли это соотношение адекватным ожидаемому или какая-то из групп необоснованно перепредставлена. Именно расчет ожидаемого соотношения полицейских остановок в разбивке по расам представляет собой основную аналитическую сложность.

Схема, разработанная Дж. Ламбертом в середине 90-х годов, была достаточно надежна, однако чрезвычайно трудоемка, дорогостояща и фактически не доступна для большинства исследовательских групп при отсутствии соответствующего финансирования (Harris 1999; Walker 2001). И даже когда научный коллектив имел возможность проводить включенные наблюдения, они были ограничены небольшим количеством мест, выбираемых неслучайным образом, а значит, полученные результаты не могли быть обобщены на другие районы или дороги, где наблюдений не проводилось (Alpert, Smith, Dunham 2004).

Альтернативный подход был предложен Д. Харрисом в 1999 г. и опробован в его исследовании, проведенном по заказу Генеральной Ассамблеи штата Огайо. Установив долевое соотношение штрафов ${ }^{10}$, выписанных черным и белым автомобилистам в четырех муниципалитетах Огайо, Д. Харрис использовал в качестве знаменателя данные переписи населения, скорректировав их по возрасту ${ }^{11}$ и расовому распределению домохозяйств, владеющих автомобилями (Harris 1999). Однако, несмотря на кажущуюся логичность предложенной модели, она страдала массой неустранимых недостатков:

\footnotetext{
${ }^{10}$ Информация о штрафах была использована Д. Харрисом вследствие отсутствия информации об остановках водителей с разбивкой по расам.

${ }^{11}$ В расчет были включены только граждане, находящиеся в «возрасте вождения» (от 15 до 75 лет).
} 
во-первых, в отличие от исследования Дж. Ламберта, не была учтена возможность межрасовых различий в манере вождения, и в том числе в частоте нарушений ПДД; вовторых, данные переписи населения, даже скорректированные по возрасту и уровню владения автомобилями, не давали надежной информации обо всех участниках дорожного движения. В частности, эти данные не учитывали жителей соседних городов, которые проезжали по дорогам исследуемых муниципалитетов, и недокументированных граждан, не охватываемых переписью. Кроме того, данные переписи измеряли статические группы населения (домохозяйства), тогда как остановки водителей и пассажиров касались временного населения, которое перемещалось разными способами и разными путями (Lamberth 2004) $^{12}$. В итоге уже в начале 2000-х годов среди специалистов по расовому профилированию установился консенсус относительно ненадежности использования данных переписи населения ${ }^{13}$ в качестве контрольного показателя (Walker 2001; Lamberth 2004; Engel, Calnon 2004; Alpert, Smith, Dunham 2004).

В 2004 г. Дж. Альперт, М. Смит и Р. Данхэм предложили использовать данные об авариях для оценки расового состава участников дорожного движения. Опираясь на результаты исследований о ДТП, авторы пришли к выводу, что граждане, попавшие в аварию, но не являвшиеся её виновниками, представляют собой случайную выборку вождения. Поскольку стать участником ДТП не по своей вине может каждый, расовый состав невиновных участников аварий должен более или менее объективно отражать расовый состав водителей и может быть использован в качестве «знаменателя» (Alpert, Smith, Dunham 2004). Впрочем, и этот метод имел очевидные ограничения: разбивка данных об участниках ДТП по конкретным местам, времени и дням недели была затруднена вследствие относительно небольшого числа аварий, а значит, сравнение было возможно только на высоком уровне агрегирования и подходило далеко не ко всем исследованиям расового профилирования (Engel, Calnon 2004).

В отсутствие возможности проводить включенное наблюдение проблематичной становилась и оценка межрасовых различий в частоте нарушения правил дорожного движения. Некоторые полицейские управления в своих отчетах пытались рассчитать индексы преступности для представителей разных рас, однако все эти попытки проваливались по двум причинам. Во-первых, такие индексы нередко составлялись на основе агрегированных показателей преступности (а не нарушений ПДД), что явно выходило за рамки поведения на дорогах и не являлось аналитически обоснованным (Alpert, Smith, Dunham 2004). Во-вторых, статистика преступности в случае наличия расовой дискриминации в системе уголовной юстиции, могла отражать не реальную картину, а результат работы расово предвзятых сотрудников правоохранительных органов (Walker 2001).

\footnotetext{
12 Исследование английской полиции, проведенное в 2000 г., эмпирически подтвердило предположение о том, что «показатели постоянного населения существенно отличаются от показателей населения, фактически доступного для остановки» (MVA, Miller 2000: III).

${ }^{13}$ Аналогичные неустранимые ограничения были выявлены и при использовании данных о гражданах, имеющих водительские права.
} 
Предположив, что проблемы, связанные с расчетом знаменателя ${ }^{14}$ и использованием официальных данных о преступности, могут быть непреодолимыми, С. Уолкер сделал попытку обойти их при помощи использовании систем раннего предупреждения ${ }^{15}$, существующих в полицейских управлениях. Идея С. Уолкера состояла в том, чтобы посредством сравнения показателей работы полицейских, действующих в одних и тех же или аналогичных условиях, выявить сотрудников правоохранительных органов, совершающих несоразмерное количество остановок, обысков, штрафов и арестов граждан из числа меньшинств (Walker 2001). Впрочем, по признанию самого автора, этот подход тоже имел по крайней мере два серьезных ограничения. Во-первых, он мог служить только для выявления расового дисбаланса в действиях отдельных офицеров, но не целых полицейских подразделений, поскольку в случае, если расовая дискриминация была официально санкционированной, все сотрудники должны были совершать несоразмерное количество остановок и обысков представителей меньшинств. Во-вторых, доступ к персонализированной информации о работниках правоохранительных органов был чрезвычайно затруднен и необходимые данные, скорее всего, не могли быть получены исследователями.

Другой попыткой обойти сложности с расчетом знаменателя и оценкой возможных расовых различий в частоте правонарушений стал так называемый тест на результат, который заключался в сравнении продуктивности остановок и обысков белых и черных автомобилистов (Knowles, Persico, Todd 1999; Ayres 2002). Предполагалось, что, если обыски чернокожих водителей систематически менее результативны, чем обыски белых, то можно заключить, что действия полиции неоправданны и предвзяты. Несмотря на популярность теста на результат, он также не был лишен ряда серьезных недостатков (Engel 2008). Главным и потенциально непреодолимым ограничением теста являлось лежащее в его основе допущение, что полицейские действуют абсолютно рационально и обладают всей полнотой информации, позволяющей им максимизировать свою полезность, что, очевидно, не соответствовало действительности.

Решение перечисленных методологических проблем не найдено до сегодняшнего дня. По этой причине наиболее надежным способом установления расового дисбаланса в полицейских остановках и обысках автомобилистов и пешеходов считается сочетание двух и более аналитических подходов.

\footnotetext{
14 Расчет знаменателя, как говорилось выше, представляет собой попытку вычисления ожидаемого соотношения непредвзятых полицейских остановок и обысков граждан в разбивке по расам для последующего сравнения этого гипотетического соотношения с реальным. Как правило, подобные расчеты пытаются установить либо долевое соотношение доступных для проверки черных и белых автомобилистов/пешеходов, либо ожидаемую частоту совершения правонарушений гражданами разных рас, либо оба этих показателя.

${ }^{15}$ Системы раннего предупреждения (EW) служат для выявления сотрудников с «проблемным» поведением, в частности тех, на которых особенно часто поступают жалобы от граждан.
} 


\section{ПРОБЛЕМЫ ТЕОРЕТИЧЕСКОЙ ИНТЕРПРЕТАЦИИ РАСОВОГО ДИСБАЛАНСА В ПОЛИЦЕЙСКОЙ ДЕЯТЕЛЬНОСТИ}

Помимо методологических сложностей в сборе и анализе данных, существуют не менее серьезные проблемы в их теоретической интерпретации. В 2004 г. Р. Энгель, Дж. Калнон и Т. Бернард в статье «Теория и расовое профилирование: недостатки и будущие направления исследований» убедительно показали, что работы по расовому профилированию не могут считаться полноценными научными исследованиями, поскольку в них отсутствует теоретическая база (Engel, Calnon, Bernard 2004: 260). Лежащее в основе значительной части таких работ непроблематизируемое допущение, что расовый дисбаланс в действиях полиции является безусловным отражением расовой предвзятости, не выдерживает критики и полностью игнорирует другие, в том числе расово нейтральные, объяснения.

Сходные соображения были высказаны относительно исследований общественного мнения, авторы которых на основании суждений респондентов о правоохранительных органах делали выводы о работе полиции и о расово дифференцированном обращении полицейских с гражданами (Liu, Crank 2010). Подчеркивая тот факт, что изучение мнений о правоохранительных органах способно больше сказать о самих респондентах, чем о полиции, Я. Лю и Дж. Крэнк призвали отказаться от некритичного отождествления того, как люди оценивают полицейскую деятельность, с тем, какой она является на самом деле.

Вопрос о существовании расового профилирования, таким образом, не может быть окончательно разрешен, исходя из простого установления расового дисбаланса в правоохранительной деятельности или расовых различий в оценках правоохранительных органов.

Существует по меньшей мере два концептуальных подхода к объяснению дифференцированного воздействия полиции на меньшинства, не предполагающих обязательного наличия расовой дискриминации. Первый из них это так называемая «экологическая концепция». Авторы, придерживающиеся данного подхода, подчеркивают значение географического распределения полицейских сил и концентрации меньшинств в неблагополучных районах, что может обусловливать расовые диспропорции в количественных показателях правоохранительной деятельности (MVA, Miller 2000; Petrocelli, Piquero, Smith 2003; Terril, Reisig 2003; Weitzer 2010).

Вторая концепция, иногда называемая «интеракционистской», фокусирует внимание на особенностях поведения граждан из числа меньшинств, которые могут делать их подозрительными или неприятными в глазах полицейских. В работах авторов этого направления указывается, что расовые диспропорции в полицейской деятельности могут быть связаны с тем, что меньшинства чаще демонстрируют неуважение по отношению к сотрудникам правоохранительных органов (Engel, Sobol, Worden 2000; Reisig et al. 2004) или ведут себя «подозрительным» ${ }^{16}$ образом (Engel 2008).

\footnotetext{
${ }^{16}$ Под «подозрительным» поведением подразумевается ряд невербальных поведенческих реакций, которые полицейских учат распознавать как указания на неискренность подозреваемых. Например, избегание зрительного контакта, частые неуместные улыбки, беспокойные движения рук, нервозность и др.
} 
Объяснительные модели собственно расового профилирования включают в себя по крайней мере четыре различных направления. Дж. Ли разработал удачную классификацию, охватывающую большинство из них, предложив расположить все имеющиеся подходы на континууме двух полярностей: индивидуальная/институциональная и интенциональная/непроизвольная расовая избирательность (Lea 1986).

Подход, согласно которому причину расового профилирования следует искать в индивидуальном поведении отдельных офицеров полиции, называют концепцией «гнилого яблока». Авторы, работающие в рамках этого направления, акцентируют внимание на расовых предрассудках и негативных личностных характеристиках некоторых сотрудников правоохранительных органов (Donner 2013; Carton et al. 2016). Концепция «Гнилого яблока» неоднократно подвергалась критике за то, что она сводит проблему неправомерного поведения полицейских (в частности, расового профилирования) к проступкам и личностным особенностям отдельных сотрудников полиции, оставляя за кадром организационные и институциональные факторы, которые могут его обусловливать (Bonanno 2015).

Альтернативной объяснительной моделью служит концепция «полицейской субкультуры». Она связывает расовое профилирование со спецификой отбора и процесса профессионализации сотрудников правоохранительных органов. Сторонники этой концепции отмечают, что в полицию набирают людей с консервативными и авторитарными взглядами, которые со временем ещё более укрепляются под воздействием профессиональной субкультуры (Colman, Gorman 1982; Twersky-Glasner 2005; Gatto et al. 2009; Lynch 2018). Утверждается, что полицейские как профессиональная группа характеризуются повышенным цинизмом, подозрительностью, предвзятостью, мачизмом, закрытостью и круговой порукой (Griffin, Ruiz 1999; Bannish, Ruiz 2003). Критики концепции «полицейской субкультуры» указывают на то, что многие сторонники данного подхода серьезно упрощают реальную картину и негативно стереотипизируют полицейских (Waddington 1999; Paoline, Myers, Worden 2000; Reiner 2016). Как справедливо замечает П. Веддингтон «соблазнительно легко приписать плохие результаты плохим людям» (Waddington 1999), однако подобное объяснение вряд ли может быть признано удовлетворительным, особенно в научном дискурсе.

Ещё одним подходом к объяснению расового профилирования является концепция институционального расизма. Она предполагает, что причины расовой предвзятости полиции следует искать в расовых предрассудках, глубоко укоренившихся в обществе в целом (Hollinsworth 1992; Holdaway, O’Neill 2007; Correll et al. 2014), или в дискриминационной государственной политике, яркими примерами которой могут служить войны с наркотиками и терроризмом (Chambliss 1994; Ramirez, Hoopes, Quinlan 2003; Fiala 2003; Bah 2006; Torres, Shahshahani, Tavaras 2015). Критики институциональной концепции отмечают, что поиск источников расового профилирования в неявных предрассудках, распространенных в обществе, обладает внутренней противоречивостью и ограниченной аналитической полезностью, а утверждения о намеренно дискриминационной государственной политике зачастую чрезмерно политизированы и лишены убедительных эмпирических доказательств (Anthias 1999; Phillips 2011). 
Исследования авторов, работающих в трех перечисленных направлениях, как правило, можно легко разместить на обоих континуумах, предложенных Дж. Ли. Так, очевидно, что концепция «гнилого яблока» отражает индивидуальную перспективу, тогда как концепции «полицейской субкультуры» и «институционального расизма» фокусируются на институциональных аспектах расовой дискриминации. Кроме того, в рамках каждого из этих направлений можно обнаружить работы, где анализируется не только явная расовая предвзятость, но и подсознательные предрассудки, которые могут обусловливать расовое профилирование (Eberhardt et al. 2004; Smith, Alpert 2007; Satzewich, Shaffir 2009).

Однако существует как минимум одна объяснительная модель расовой предвзятости в полицейской деятельности, не укладывающаяся в классификацию Дж. Ли. Согласно этому подходу причина расового профилирования заключается в повышенной склонности отдельных меньшинств к совершению преступлений, а само профилирование представляет собой рациональную полицейскую стратегию, обусловленную ограниченностью ресурсов (Mac Donald 2001; 2002). Критики данного подхода подчеркивают противоречивость результатов эмпирических исследований, которые не позволяют подтвердить или опровергнуть тезис о расовых различиях в частоте правонарушений: в то время как некоторые авторы обнаруживают подобные различия (Lange, Johnson, Voas 2005; Mosher et al. 2008), другие - нет (Horton 2002; Lamberth 2004). Кроме того, критики справедливо указывают, что даже если межрасовые различия в частоте правонарушений существуют, основополагающий принцип равенства перед законом требует, чтобы с невинными гражданами любой расы обращались одинаково, а значит, расовое профилирование не может рассматриваться как допустимая правоохранительная стратегия (Thacher 2002; Harcourt 2004).

\section{Выводы}

Изучение расового профилирования - сравнительно новая, быстро развивающаяся область исследований, возникшая в англоязычном научном мире и распространившаяся далеко за его пределы. Несмотря на растущую популярность этого исследовательского направления, его разработка затруднена рядом методологических и теоретических проблем. Главными из них являются сложность эмпирического обнаружения расового дисбаланса в работе правоохранительных органов, связанная с продолжающимися поисками надежного «знаменателя», и недостаточность простого установления расовых диспропорций для однозначного заключения о наличии расового профилирования. Вследствие того, что в значительной части исследований указанные ограничения не были учтены, а выводы были слишком сильными и поспешными, их использование должно быть крайне осторожным ${ }^{17}$. Признание существующих ограничений при сборе и анализе количественных данных о расовом дисбалансе в полицейской деятельности тем более важно, что опубликование

\footnotetext{
${ }^{17}$ Некоторые специалисты даже полагают, что результаты значительной части исследований расового профилирования не заслуживают доверия и бесполезны с практической точки зрения (Engel 2008; Liu, Crank 2010).
} 
результатов таких исследований нередко имеет политические последствия и может привести к деградации взаимодействия общественности с правоохранительными органами.

Чтобы не повторять распространенных ошибок, допущенных на раннем этапе изучения расового профилирования, при планировании исследования следует учитывать накопленный опыт. И хотя к настоящему моменту универсальный рецепт успешного изучения расового профилирования ещё не создан, некоторые рекомендации можно сделать уже сейчас.

- При разработке исследовательского дизайна, выборе теоретической парадигмы и интерпретации полученных эмпирических данных следует четко определиться с объектом исследования. Не стоит смешивать намеренное расовое профилирование, санкционированное государством; политику отдельных полицейских управлений, не связанную с государственной политикой; инициативу отдельных сотрудников полиции; а также статистическую дискриминацию, являющуюся отражением социального неравенства.

- При сборе и анализе количественной информации необходимо использовать несколько источников данных и несколько аналитических методов. Это позволит сравнить полученные результаты и повысить надежность выводов.

- Не стоит делать поспешных заключений. Даже если полученные данные укладываются в избранную исследователем концептуальную рамку, необходимо рассмотреть все существующие альтернативные объяснения, в том числе расово нейтральные.

\section{ЛИТЕРАТУРА}

Воронков В., Гладарев Б., Сагитова Л. (Ред.) (2011). Милиция и этнические мигрантыл: практики взаимодействия. СПб.: Алетейя. 638 с.

Григорьева К.С. (2019). Этническая дискриминация в борьбе с преступностью и терроризмом - вопрос здравого смысла? Социологическое обозрение, 18(1), 107-139. URL: https://sociologica.hse.ru/data/2019/03/28/1187304505/SocOboz_18_1_107139_Grigoryeva.pdf

ЕКРН (2004). Общеполитическая рекомендащчи Европейской комиссии по борьбе с расизмом и нетерпимостью №8: «По борьбе с расизмом в проиессе противодействия терроризму». URL: https://rm.coe.int/compilation-of-ecri-s-general-policyrecommendations-march-2018-russia/1680923e0a

ЕКРН (2007). Общееполитическая рекомендация Европейской комиссии по борьбе с расизмом и нетерпимостью №11: «О борьбе с расизмом и расовой дискриминацией 8 работе правоохранительных органов». URL: https://rm.coe.int/compilation-of-ecri-sgeneral-policy-recommendations-march-2018-russia/1680923e0a

Этнически избирательньй подход в действиях милищии в московском метро (2006). М.: Новая юстиция.

Alpert G.P., Smith M.R., Dunham R.G. (2004). Toward a better benchmark: Assessing the utility of not-at-fault traffic crash data in racial profiling research. Justice Research and Policy, 6(1), 43-70. DOI: 10.3818/JRP.6.1.2004.43 
Anthias F. (1999). Institutional Racism, Power and Accountability. Sociological Research Online, 4(1). DOI: 10.5153/sro.239. URL:

http://www.socresonline.org.uk/4/lawrence//anthias.html

Ayres I. (2002). Outcomes tests of racial disparities in police practices. Justice Research and Policy, 4 (Special Issue), 131-142. URL:

https://pdfs.semanticscholar.org/19b3/ad37064fb5d4a32314bb9ba0e613e180f350.pdf

Bah A.B. (2006). Racial Profiling and the War on Terror: Changing Trends and Perspectives. Ethnic Studies Review, 29, 76-100. DOI: 10.1525/esr.2006.29.1.76

Bannish H., Ruiz J. (2003). The Antisocial Police Personality: A View from the Inside. International Journal of Public Administration, 26(7), 831-881. DOI: 10.1081/PAD120019322

Bittner E. (1970). The Functions of the Police in Modern Society. Washington: U.S. Government Printing Office.

Bonanno E.R. (2015). An Evidential Review of Police Misconduct: Officer versus Organization. 2015 Undergraduate Awards, 9. URL: https://ir.lib.uwo.ca/ungradawards_2015/9

Carton S., Helsby J., Joseph K., Mahmud A., Park Y., Walsh J., Cody C.,

Patterson E., Haynes L., Ghani R. (2016, August). Identifying Police Officers at Risk of Adverse Events. In Proceedings of the 22nd ACM SIGKDD International Conference on Knowledge Discovery and Data Mining (pp. 67-76). ACM. DOI: 10.1145/2939672.2939698.

Chambliss W.J. (1994). Another Lost War: The Costs and Consequences of Drug Prohibition. Social Justice, 22(2), 101-125. URL: https://www.jstor.org/stable/29766881

Chambliss W.J., Nagasawa R.H. (1969). On the Validity of Official Statistics A Comparative Study of White, Black, and Japanese High-School Boys. Journal of Research in Crime and Delinquency, 6(1), 71-77. DOI: 10.1177/002242786900600108

Colb S.F. (2004). Profiling with Apologies. Ohio State Journal of Criminal Law, 1(2), 611-625. URL: https://scholarship.law.cornell.edu/cgi/viewcontent.cgi?article=1803\&context=facpub

Colman A.M., Gorman L.P. (1982). Conservatism, Dogmatism, and Authoritarianism in British Police Officers. Sociology, 16(4), 1-11. doi:10.1177/0038038582016001004

Correll J., Hudson S.M., Guillermo S., Ma D.S. (2014). The Police Officer's Dilemma: A Decade of Research on Racial Bias in the Decision to Shoot. Social and Personality Psychology Compass, 8(5), 201-213. doi:10.1111/spc3.12099

Dearden L. (2017). More than half British people support racial profiling of Muslims and Arabs for security reasons, survey reveals. Independent. URL:

https://www.independent.co.uk/news/uk/home-news/racial-profiling-british-people-muslimsarabs-support-security-anti-terrorism-attacks-survey-caabu-a7966666.html

Donner C.M. (2013). Examining the link between self-control and misconduct in a multi-agency sample of police supervisors: A test of two theories. (Doctoral dissertation). Retrieved from Graduate Theses and

Dissertations. URL: http://scholarcommons.usf.edu/etd/4470

Eberhardt J.L., Goff Ph.A., Purdie V.J., Davies P.G. (2004). Seeing Black: Race, Crime, and Visual Processing. Journal of Personality and Social Psychology, 87(6), 876-893.

DOI: $10.1037 / 0022-3514.87 .6 .876$ 
Engel R.S. (2008). A Critique of the "Outcome Test" in Racial Profiling Research. Justice Quarterly, 25(1), 1-36. DOI: 10.1080/07418820701717177

Engel R.S., Calnon J.M. (2004). Comparing Benchmark Methodologies for Police-Citizen Contacts: Traffic Stop Data Collection for the Pennsylvania State Police. Justice Quarterly, 7(1), 97-125. doi:10.1177/1098611103257686

Engel R.S., Calnon J.M., Bernard T.J. (2004). Theory and Racial Profiling: Shortcomings and Future Directions in Research. Justice Quarterly. 19(2), 249-273.

DOI: $10.1080 / 07418820200095231$

Engel R.S., Sobol J.J., Worden R.E. (2000). Further exploration of the demeanor hypothesis: The interaction effects of suspects' characteristics and demeanor on police behavior. Justice Quarterly, 17(2), 235-258. doi: 10.1080/07418820000096311

Fiala I.J. (2003). Anything new? The racial profiling of terrorists. Criminal Justice Studies: A Critical Journal of Crime, Law and Society, 16(1), 53-58. doi:10.1080/08884310309610

FRA (2010). EU-MIDIS: European Union Minorities and Discrimination Survey: Main Results Report. Luxembourg: Publications Office of the European Union. Retrieved from URL: https://fra.europa.eu/en/publication/2012/european-union-minorities-and-discriminationsurvey-main-results-report

Gatto J., Dambrun M., Kerbrat C., de Oliveira P. (2009). Prejudice in the police: On the processes underlying the effects of selection and group socialization. European Journal of Social Psychology, 40(2), 252 - 269. doi:10.1002/ejsp.617

Gilroy P. (1982). The myth of black criminality. Socialist Register, 47-56. URL: https://socialistregister.com > article > download

Goldstein H. (1963). Police Discretion: The Ideal Versus the Real. Public Administration Review, XXIII(3), 140-148. URL: https://papers.ssrn.com/sol3/papers.cfm?abstract_id=2537432

Goris I., Jobard F., Lévy R. (2009). Police et minorités visibles: les contrôles d'identité à Paris. New York: Open Society Institute.

Griffin C., Ruiz J. (1999). The Sociopathic Police Personality: Is It a Product of the "Rotten Apple" or the "Rotten Barrel"? Journal of Police and Criminal Psychology, 14(1), 28-37. DOI: $10.1007 / \mathrm{BF} 02813860$

Harcourt B.E. (2004). Rethinking Racial Profiling: A Critique of the Economics, Civil Liberties, and Constitutional Literature, and of Criminal Profiling More Generally. University of Chicago Law Review, 71(4), 1275 - 1381. DOI: 10.2139/ssrn.471901

Harris D.A. (1999). The Stories, the Statistics, and the Law: Why "Driving While Black" Matters. Minnesota Law Review, 84(2), 265-326. URL: https://ssrn.com/abstract=199508

Holdaway S., O'Neill M. (2007). Where has all the racism gone? Views of racism within constabularies after Macpherson. Ethnic and Racial Studies, 30(3), 397-415.

DOI: $10.1080 / 01419870701217480$

Hollinsworth D. (1992). Cultural awareness training, racism awareness training or antiracism?: Strategies for combating institutional racism. Journal of Intercultural Studies, 13(2), 37-52. DOI: $10.1080 / 07256868.1992 .9963389$

Horton A. (2002). Violent Crimes and Racial Profiling. Journal of Human Behavior in the Social Environment, 6(4), 87-106. doi:10.1300/J137v06n04_05 
Jones J.M. (2001). The Impact of the Attacks on America. Gallup. URL: https://news.gallup.com/poll/4894/impact-attacks-america.aspx

Knowles J., Persico N., Todd P. (1999). Racial Bias in Motor Vehicle Searches: Theory and Evidence. The Journal of Political Economy, 109(1), 203-229. URL: https://www.nber.org/papers/w7449.pdf

LaFave W.R. (1962). Detention for Investigation by the Police: An Analysis of Current Practices. Washington University Law Review, 1962(3), 331-399. URL: https://openscholarship.wustl.edu/law_lawreview/vol1962/iss3/6

Lamberth J. (2004). Ann Arbor Police Department Traffic Stop Data Collection Methods and Analysis Study: Report for the City of Ann Arbor. Retrieved from URL: http://annarborchronicle.com/wpcontent/uploads/2012/07/A2_FinalReport_012204_v6co.pdf

Lange J.E., Johnson M.B., Voas R.B. (2005). Testing the racial profiling hypothesis for seemingly disparate traffic stops on the New Jersey Turnpike. Justice Quarterly, 22(2), 193223. DOI: $10.1080 / 07418820500088952$

Lea J. (1986). Police racism: some theories and their policy Implications. In Matthews R. Young J. (Eds.), Confronting Crime (pp. 145-165). London: Sage Publications. URL: https://www.academia.edu/26225451/Police_racism_some_theories_and_their_policy_implic ations_1986_

Liu Ya., Crank J. (2010). Attitudes toward the police: a critique and recommendations. Criminal Justice Studies, 23(2), 99-117. doi:10.1080/1478601X.2010.485454

Lynch C.G. (2018). Don't let them kill you on some dirty roadway: survival, entitled violence, and the culture of modern American policing. Contemporary Justice Review, 21(1), 33-43. doi:10.1080/10282580.2018.1415045

Mac Donald H. (2001). The Myth of Racial Profiling. City Journal. URL: https://www.cityjournal.org/html/myth-racial-profiling-12022.html

Mac Donald H. (2002). The Racial Profiling Myth Debunked. City Journal. URL: https://www.city-journal.org/html/racial-profiling-myth-debunked-12244.html

Miller J., Gounev Ph., Pap A.L., Wagman D., Balogi A., Bezlov T., Simonovits B., Vargha L. (2008). Racism and Police Stops: Adapting US and British Debates to Continental Europe. European Journal of Criminology, 5(2), 161-189. DOI: 10.1177/1477370807087641

Mosher C., Pickerill J.M., Pratt T., Lovrich N. (2008). The importance of context in understanding biased policing: state patrol traffic citations in Washington State. Police Practice and Research: An International Journal, 9(1), 43-57.

DOI: $10.1080 / 15614260801969920$

MVA, Miller J. (2000). Profiling Populations Available for Stops and Searches. Police Research Series Paper 131. London: Home Office. Retrieved from https://www.semanticscholar.org/paper/Profiling-populations-available-for-stops-andMiller/1005d76d353c62fcd4bf334cfd6068079f09b13d

Paoline E.A., Myers S.M., Worden R.E. (2000). Police Culture, Individualism, and Community Policing: Evidence from Two Police Departments. Justice Quarterly, 17(3), 575-605. DOI: $10.1080 / 07418820000094671$

Pap A.L. (2011). Ethnic Profiling and Discrimination: The International Context and Hungarian Empirical Research Findings. Acta Juridica Hungarica, 52(4), 273-295.

DOI: 10.1556/AJur.52.2011.4.1 
Petrocelli M., Piquero A.R., Smith M.R. (2003). Conflict theory and racial profiling: An empirical analysis of police traffic stop data. Journal of Criminal Justice, 31(1), 1-11. DOI: $10.1016 / \mathrm{S} 0047-2352(02) 00195-2$

Phillips C. (2011). Institutional racism and ethnic inequalities: an expanded multilevel framework. Journal of social policy, 40(1), 173-192. DOI: 10.1017/S0047279410000565

Posner R.A. (2006). Not a Suicide Pact: The Constitution in a Time of National Emergency. New York: Oxford University Press.

Quinney R. (1970). The Social Reality of Crime. Boston, MA: Little, Brown.

Ramirez D.A., Hoopes J., Quinlan T.L. (2003). Defining Racial Profiling in a Post-September 11 World. American Criminal Law Review, 40(3), 1195-1233.

Reiner R. (2016). Is police culture cultural? Policing: a Journal of Policy and Practice, 11(3), 236-241. DOI: 10.1093/police/paw046

Reisig M.D., McCluskey J.D., Mastrofski S.D., Terrill W. (2004). Suspect disrespect toward the police. Justice Quarterly, 21(2), 241-268. DOI: 10.1080/07418820400095801

Remington F.J. (1960). Law Relating to on the Street Detention, Questioning and Frisking of Suspected Persons and Police Arrest Privileges in General. Journal of Criminal Law and Criminology, 51(4), 386-394. URL:

https://scholarlycommons.law.northwestern.edu/cgi/viewcontent.cgi $?$ article $=4963 \&$ context $=\mathrm{j}$ clc

Satzewich V., Shaffir W. (2009). Racism versus Professionalism: Claims and Counter-claims about Racial Profiling. Canadian Journal of Criminology and Criminal Justice, 51(2), 199226. DOI: $10.3138 /$ cjecj.51.2.199

Sherman L.W. (1980). Causes of Police Behavior: The Current State of Quantitative Research. Journal of Research in Crime and Delinquency, 17(1), 69-100. DOI: $10.1177 / 002242788001700106$

Skolnick J.H. (1966). Justice Without Trial: Law Enforcement in Democratic Society. New York: John Wiley \& Sons.

Smith M.R., Alpert G.P. (2007). Explaining Police Bias: A Theory of Social Conditioning and Illusory Correlation. Criminal Justice and Behavior, 34(10), 1262 -1283.

DOI: $10.1177 / 0093854807304484$

State v. Soto. (1966). 324 N.J. Super. 66 (N.J. Super. App. Div. 1996).

Terrill W., Reisig M.D. (2003). Neighborhood Context and Police Use of Force. Journal of Research in Crime and Delinquency, 40(3), 291-321. DOI: 10.1177/0022427803253800

Thacher D. (2002). From racial profiling to racial inequality: Rethinking equity in police stops and searches. Working paper, Ford School of Public Policy, University of Michigan. URL: http://fordschool.umich.edu/research/papers/PDFfiles/02-006.pdf

Torres C., Shahshahani A., Tavaras T. (2015). Indiscriminate Power: Racial Profiling and Surveillance Since 9/11. University of Pennsylvania Journal of Law and Social Change, 18(4), 283-310. URL: https://scholarship.law.upenn.edu/jlasc/vol18/iss4/1

Twersky-Glasner A. (2005). Police personality: What is it and why are they like that? Journal of Police and Criminal Psychology, 20(1), 56-67. 
Waddington P.A.J. (1999). Discretion, 'Respectability' and Institutional Police Racism.

Sociological Research Online, 4(1). URL:

http://www.socresonline.org.uk/4/lawrence/waddington.html

Walker S. (1992). Origins of the contemporary criminal justice paradigm: The American bar foundation survey, 1953-1969. Justice Quarterly, 9(1), 47-76. doi:

$10.1080 / 07418829200091251$

Walker S. (2001). Searching for the Denominator: Problems with Police Traffic Stop Data and an Early Warning System Solution. Justice Research and Policy, 3(1), 63-95. doi:10.3818/JRP.3.1.2001.63

Weitzer R. (2010). Race and Policing in Different Ecological Contexts. In Rice S.K. (Ed.), Race, Ethnicity, and Policing: New and Essential Readings, (pp. 118-139). New York: New York University Press.

Wilkins v. Maryland State Police (1994). Civ. No. MJG-93-468 USDC MD. 


\title{
RACIAL PROFILING: \\ THE HISTORY AND CURRENT STATE OF RESEARCH
}

\author{
KSENIJA GRIGORYEVA
}

\begin{abstract}
Studies of racial profiling in Russia have confirmed the existence of this problem. However, its development, which began in the mid-2000s, has so far progressed only slightly. The Russian-speaking reader is practically unfamiliar with the theoretical and methodological approaches to the study of racial profiling developed abroad. This article is intended to fill the lack of information about foreign studies of racial profiling and is the first review of the literature on this topic in Russian. The article examines the history of the study of racial discrimination in the criminal justice system in the English-speaking scientific world, and analyzes the transformation of racial profiling into a separate research area in the early nineties and its further development in the 2000s. The main methodological approaches to the study of racial profiling, their possibilities and limitations are presented. The key theoretical approaches to the understanding of racial disparities in law enforcement are considered. In conclusion, based on the understanding of the experience gained, a number of recommendations for planning future studies are given. Attention is drawn to the need to distinguish more clearly between intentional racial profiling authorized by the state; the policies of individual police departments; the personal initiative of police officers; and statistical discrimination reflecting social inequality. When conducting quantitative studies, it is recommended to use several data sources and several analytical methods. The need to be cautious in conclusions, avoiding haste and bias, is emphasized. When interpreting the empirical data obtained, it is recommended to consider all possible conceptual explanations, including racially neutral ones.
\end{abstract}

Key words: racial discrimination, criminal justice, racial profiling, the denominator problem, individual and institutional racial bias, conscious and unconscious racial prejudices.

KSENIJA GRIGORYEVA (kseniagrigoryeva@yandex.ru), InSTITUTE OF SOCIOLOgy, FEDERAL CENTER OF THEORETICAL AND APPLIED SOCIOLOGY, RUSSIAN ACADEMY OF SCIENCES, RUSSIA.

DATE RECEIVED : OCTOBER 2019.

\section{REFERENCES}

Alpert G.P., Smith M.R., Dunham R.G. (2004). Toward a better benchmark: Assessing the utility of not-at-fault traffic crash data in racial profiling research. Justice Research and Policy, 6(1), 43-70. DOI: 10.3818/JRP.6.1.2004.43

Anthias F. (1999). Institutional Racism, Power and Accountability. Sociological Research Online, 4(1). DOI: 10.5153/sro.239. URL: http://www.socresonline.org.uk/4/lawrence/anthias.html

Ayres I. (2002). Outcomes tests of racial disparities in police practices. Justice Research and Policy, 4 (Special Issue), 131-142. URL: https://pdfs.semanticscholar.org/19b3/ad37064fb5d4a32314bb9ba0e613e180f350.pdf

Bah A.B. (2006). Racial Profiling and the War on Terror: Changing Trends and Perspectives. Ethnic Studies Review, 29, 76-100. doi: 10.1525/esr.2006.29.1.76

Bannish H., Ruiz J. (2003). The Antisocial Police Personality: A View from the Inside. International Journal of Public Administration, 26(7), 831-881. DOI: 10.1081/PAD120019322 
Bittner E. (1970). The Functions of the Police in Modern Society. Washington: U.S. Government Printing Office.

Bonanno E.R. (2015). An Evidential Review of Police Misconduct: Officer versus Organization. 2015 Undergraduate Awards, 9. URL: https://ir.lib.uwo.ca/ungradawards_2015/9

Carton S., Helsby J., Joseph K., Mahmud A., Park Y., Walsh J., Cody C.,

Chambliss W.J. (1994). Another Lost War: The Costs and Consequences of Drug Prohibition. Social Justice, 22(2), 101-125. URL: https://www.jstor.org/stable/29766881

Chambliss W.J., Nagasawa R.H. (1969). On the Validity of Official Statistics A Comparative Study of White, Black, and Japanese High-School Boys. Journal of Research in Crime and Delinquency, 6(1), 71-77. DOI: 10.1177/002242786900600108

Colb S.F. (2004). Profiling with Apologies. Ohio State Journal of Criminal Law, 1(2), 611-625. URL: https://scholarship.law.cornell.edu/cgi/viewcontent.cgi?article=1803\&context=facpub

Colman A.M., Gorman L.P. (1982). Conservatism, Dogmatism, and Authoritarianism in British Police Officers. Sociology, 16(4), 1-11. DOI: 10.1177/0038038582016001004

Correll J., Hudson S.M., Guillermo S., Ma D.S. (2014). The Police Officer's Dilemma: A Decade of Research on Racial Bias in the Decision to Shoot. Social and Personality Psychology Compass, 8(5), 201-213. DOI: 10.1111/spc3.12099

Dearden L. (2017). More than half British people support racial profiling of Muslims and Arabs for security reasons, survey reveals. Independent. URL:

https://www.independent.co.uk/news/uk/home-news/racial-profiling-british-people-muslimsarabs-support-security-anti-terrorism-attacks-survey-caabu-a7966666.html

Dissertations. URL: http://scholarcommons.usf.edu/etd/4470

Donner C.M. (2013). Examining the link between self-control and misconduct in a multi-agency sample of police supervisors: A test of two theories. (Doctoral dissertation). Retrieved from Graduate Theses and

Eberhardt J.L., Goff Ph.A., Purdie V.J., Davies P.G. (2004). Seeing Black: Race, Crime, and Visual Processing. Journal of Personality and Social Psychology, 87(6), 876-893.

DOI: $10.1037 / 0022-3514.87 .6 .876$

ECRI (2004). ECRI General Policy Recommendation N8 on combating racism while fighting terrorism. Strasbourg: Council of Europe. URL: https://www.coe.int/en/web/europeancommission-against-racism-and-intolerance/recommendation-no.8

ECRI (2007). ECRI General Policy Recommendation N11 on combating racism and racial discrimination in policing. Strasbourg: Council of Europe. URL:

https://www.coe.int/en/web/european-commission-against-racism-andintolerance/recommendation-no.11

Engel R.S. (2008). A Critique of the "Outcome Test" in Racial Profiling Research. Justice Quarterly, 25(1), 1-36. DOI: 10.1080/07418820701717177

Engel R.S., Calnon J.M. (2004). Comparing Benchmark Methodologies for Police-Citizen Contacts: Traffic Stop Data Collection for the Pennsylvania State Police. Justice Quarterly, 7(1), 97-125. DOI: 10.1177/1098611103257686

Engel R.S., Calnon J.M., Bernard T.J. (2004). Theory and Racial Profiling: Shortcomings and Future Directions in Research. Justice Quarterly. 19(2), 249-273.

DOI: $10.1080 / 07418820200095231$ 
Engel R.S., Sobol J.J., Worden R.E. (2000). Further exploration of the demeanor hypothesis: The interaction effects of suspects' characteristics and demeanor on police behavior. Justice Quarterly, 17(2), 235-258. DOI: 10.1080/07418820000096311

Fiala I.J. (2003). Anything new? The racial profiling of terrorists. Criminal Justice Studies: A Critical Journal of Crime, Law and Society, 16(1), 53-58. doi:10.1080/08884310309610

FRA (2010). EU-MIDIS: European Union Minorities and Discrimination Survey: Main Results Report. Luxembourg: Publications Office of the European Union. Retrieved from URL: https://fra.europa.eu/en/publication/2012/european-union-minorities-and-discriminationsurvey-main-results-report

Gatto J., Dambrun M., Kerbrat C., de Oliveira P. (2009). Prejudice in the police: On the processes underlying the effects of selection and group socialization. European Journal of Social Psychology, 40(2), 252 - 269. DOI: 10.1002/ejsp.617

Gilroy P. (1982). The myth of black criminality. Socialist Register, 47-56. URL: https://socialistregister.com > article > download

Goldstein H. (1963). Police Discretion: The Ideal Versus the Real. Public Administration Review, XXIII(3), 140-148. URL: https://papers.ssrn.com/sol3/papers.cfm?abstract_id=2537432

Goris I., Jobard F., Lévy R. (2009). Police et minorités visibles: les contrôles d'identité à Paris. New York: Open Society Institute.

Griffin C., Ruiz J. (1999). The Sociopathic Police Personality: Is It a Product of the "Rotten Apple" or the "Rotten Barrel"? Journal of Police and Criminal Psychology, 14(1), 28-37. DOI: $10.1007 / \mathrm{BF} 02813860$

Grigoryeva K. (2019). Is Ethnic Discrimination a Matter of Common Sense in the Fight against Crime and Terrorism? The Russian Sociological Review, 18(1), 107-139 (In Russ.). URL: https://sociologica.hse.ru/data/2019/03/28/1187304505/SocOboz_18_1_107139_Grigoryeva.pdf

Harcourt B.E. (2004). Rethinking Racial Profiling: A Critique of the Economics, Civil Liberties, and Constitutional Literature, and of Criminal Profiling More Generally. University of Chicago Law Review, 71(4), 1275 - 1381. DOI: 10.2139/ssrn.471901

Harris D.A. (1999). The Stories, the Statistics, and the Law: Why "Driving While Black" Matters. Minnesota Law Review, 84(2), 265-326. URL: https://ssrn.com/abstract=199508

Holdaway S., O'Neill M. (2007). Where has all the racism gone? Views of racism within constabularies after Macpherson. Ethnic and Racial Studies, 30(3), 397-415. DOI: $10.1080 / 01419870701217480$

Hollinsworth D. (1992). Cultural awareness training, racism awareness training or antiracism?: Strategies for combating institutional racism. Journal of Intercultural Studies, 13(2), 37-52. DOI: $10.1080 / 07256868.1992 .9963389$

Horton A. (2002). Violent Crimes and Racial Profiling. Journal of Human Behavior in the Social Environment, 6(4), 87-106. doi:10.1300/J137v06n04_05

Jones J.M. (2001). The Impact of the Attacks on America. Gallup. URL: https://news.gallup.com/poll/4894/impact-attacks-america.aspx

Knowles J., Persico N., Todd P. (1999). Racial Bias in Motor Vehicle Searches: Theory and Evidence. The Journal of Political Economy, 109(1), 203-229. URL: https://www.nber.org/papers/w7449.pdf 
LaFave W.R. (1962). Detention for Investigation by the Police: An Analysis of Current Practices. Washington University Law Review, 1962(3), 331-399. URL:

https://openscholarship.wustl.edu/law_lawreview/vol1962/iss3/6

Lamberth J. (2004). Ann Arbor Police Department Traffic Stop Data Collection Methods and Analysis Study: Report for the City of Ann Arbor. Retrieved from URL: http://annarborchronicle.com/wpcontent/uploads/2012/07/A2_FinalReport_012204_v6co.pdf

Lange J.E., Johnson M.B., Voas R.B. (2005). Testing the racial profiling hypothesis for seemingly disparate traffic stops on the New Jersey Turnpike. Justice Quarterly, 22(2), 193223. DOI: $10.1080 / 07418820500088952$

Lea J. (1986). Police racism: some theories and their policy Implications. In Matthews R. Young J. (Eds.), Confronting Crime (pp. 145-165). London: Sage Publications. URL: https://www.academia.edu/26225451/Police_racism_some_theories_and_their_policy_implic ations_1986_

Liu Ya., Crank J. (2010). Attitudes toward the police: a critique and recommendations. Criminal Justice Studies, 23(2), 99-117. doi:10.1080/1478601X.2010.485454

Lynch C.G. (2018). Don't let them kill you on some dirty roadway: survival, entitled violence, and the culture of modern American policing. Contemporary Justice Review, 21(1), 33-43. DOI: $10.1080 / 10282580.2018 .1415045$

Mac Donald H. (2001). The Myth of Racial Profiling. City Journal. URL: https://www.cityjournal.org/html/myth-racial-profiling-12022.html

Mac Donald H. (2002). The Racial Profiling Myth Debunked. City Journal. URL: https://www.city-journal.org/html/racial-profiling-myth-debunked-12244.html

Miller J., Gounev Ph., Pap A.L., Wagman D., Balogi A., Bezlov T., Simonovits B., Vargha L. (2008). Racism and Police Stops: Adapting US and British Debates to Continental Europe. European Journal of Criminology, 5(2), 161-189. doi: 10.1177/1477370807087641

Mosher C., Pickerill J.M., Pratt T., Lovrich N. (2008). The importance of context in understanding biased policing: state patrol traffic citations in Washington State. Police Practice and Research: An International Journal, 9(1), 43-57. DOI: $10.1080 / 15614260801969920$

MVA, Miller J. (2000). Profiling Populations Available for Stops and Searches. Police Research Series Paper 131. London: Home Office. Retrieved from https://www.semanticscholar.org/paper/Profiling-populations-available-for-stops-andMiller/1005d76d353c62fcd4bf334cfd6068079f09b13d

Paoline E.A., Myers S.M., Worden R.E. (2000). Police Culture, Individualism, and Community Policing: Evidence from Two Police Departments. Justice Quarterly, 17(3), 575-605. DOI: $10.1080 / 07418820000094671$

Pap A.L. (2011). Ethnic Profiling and Discrimination: The International Context and Hungarian Empirical Research Findings. Acta Juridica Hungarica, 52(4), 273-295.

DOI: 10.1556/AJur.52.2011.4.1

Patterson E., Haynes L., Ghani R. (2016, August). Identifying Police Officers at Risk of Adverse Events. In Proceedings of the 22nd ACM SIGKDD International Conference on Knowledge Discovery and Data Mining (pp. 67-76). ACM. DOI: 10.1145/2939672.2939698. 
Petrocelli M., Piquero A.R., Smith M.R. (2003). Conflict theory and racial profiling: An empirical analysis of police traffic stop data. Journal of Criminal Justice, 31(1), 1-11. DOI: $10.1016 / \mathrm{S} 0047-2352(02) 00195-2$

Phillips C. (2011). Institutional racism and ethnic inequalities: an expanded multilevel framework. Journal of social policy, 40(1), 173-192. doi:10.1017/S0047279410000565

Posner R.A. (2006). Not a Suicide Pact: The Constitution in a Time of National Emergency. New York: Oxford University Press.

Quinney R. (1970). The Social Reality of Crime. Boston, MA: Little, Brown.

Ramirez D.A., Hoopes J., Quinlan T.L. (2003). Defining Racial Profiling in a Post-September 11 World. American Criminal Law Review, 40(3), 1195-1233.

Reiner R. (2016). Is police culture cultural? Policing: a Journal of Policy and Practice, 11(3), 236-241. DOI: 10.1093/police/paw046

Reisig M.D., McCluskey J.D., Mastrofski S.D., Terrill W. (2004). Suspect disrespect toward the police. Justice Quarterly, 21(2), 241-268. DOI: 10.1080/07418820400095801

Remington F.J. (1960). Law Relating to on the Street Detention, Questioning and Frisking of Suspected Persons and Police Arrest Privileges in General. Journal of Criminal Law and Criminology, 51(4), 386-394. URL:

https://scholarlycommons.law.northwestern.edu/cgi/viewcontent.cgi $?$ article $=4963 \&$ context $=\mathrm{j}$ clc

Satzewich V., Shaffir W. (2009). Racism versus Professionalism: Claims and Counter-claims about Racial Profiling. Canadian Journal of Criminology and Criminal Justice, 51(2), 199226. DOI: $10.3138 /$ cjecj.51.2.199

Sherman L.W. (1980). Causes of Police Behavior: The Current State of Quantitative Research. Journal of Research in Crime and Delinquency, 17(1), 69-100. DOI: $10.1177 / 002242788001700106$

Skolnick J.H. (1966). Justice Without Trial: Law Enforcement in Democratic Society. New York: John Wiley \& Sons.

Smith M.R., Alpert G.P. (2007). Explaining Police Bias: A Theory of Social Conditioning and Illusory Correlation. Criminal Justice and Behavior, 34(10), 1262 -1283.

DOI: $10.1177 / 0093854807304484$

State v. Soto. (1966). 324 N.J. Super. 66 (N.J. Super. App. Div. 1996).

Terrill W., Reisig M.D. (2003). Neighborhood Context and Police Use of Force. Journal of Research in Crime and Delinquency, 40(3), 291-321. DOI: 10.1177/0022427803253800

Thacher D. (2002). From racial profiling to racial inequality: Rethinking equity in police stops and searches. Working paper, Ford School of Public Policy, University of Michigan. URL: http://fordschool.umich.edu/research/papers/PDFfiles/02-006.pdf

Torres C., Shahshahani A., Tavaras T. (2015). Indiscriminate Power: Racial Profiling and Surveillance Since 9/11. University of Pennsylvania Journal of Law and Social Change, 18(4), 283-310. URL: https://scholarship.law.upenn.edu/jlasc/vol18/iss4/1

Twersky-Glasner A. (2005). Police personality: What is it and why are they like that? Journal of Police and Criminal Psychology, 20(1), 56-67. 
Voronkov V., Gladarev B., Sagitova L. (Eds.) (2011). Militsiya i etnicheskiye migranty: praktiki vzaimodeystviya [Police and Ethnic Migrants: Interaction Practices]. St Petersburg: Aleteya. (In Russ.).

Waddington P.A.J. (1999). Discretion, 'Respectability' and Institutional Police Racism. Sociological Research Online, 4(1). URL: http://www.socresonline.org.uk/4/lawrence/waddington.html

Walker S. (1992). Origins of the contemporary criminal justice paradigm: The American bar foundation survey, 1953-1969. Justice Quarterly, 9(1), 47-76. DOI: $10.1080 / 07418829200091251$

Walker S. (2001). Searching for the Denominator: Problems with Police Traffic Stop Data and an Early Warning System Solution. Justice Research and Policy, 3(1), 63-95.

DOI: 10.3818/JRP.3.1.2001.63

Weitzer R. (2010). Race and Policing in Different Ecological Contexts. In Rice S.K. (Ed.), Race, Ethnicity, and Policing: New and Essential Readings, (pp. 118-139). New York: New York University Press.

Wilkins v. Maryland State Police (1994). Civ. No. MJG-93-468 USDC MD. 